\title{
Multiyear and Multi-Criteria AC Transmission Expansion Planning Model Considering Reliability and Investment Costs
}

\author{
Phillipe Vilaça Gomes, João Pedro Silva and João Tomé Saraiva \\ INESC TEC and FEUP/DEEC \\ Dept. Eng. Eletrotécnica e de Computadores da Faculdade de Engenharia da Universidade do Porto - Portugal \\ phillipe.gomes@fe.up.pt, joaopedrosilva91@gmail.com, jsaraiva@fe.up.pt
}

\begin{abstract}
One of the major concerns in Power Systems is surely related with their reliability. Long-term expansion planning studies traditionally use the well-known deterministic "N-1" contingency criterion. However, this criterion is applied based on worst-case analyses and the obtained plan may originate over-investments. Differently, probabilistic reliability approaches can incorporate different type of uncertainties that affect power systems. In this work, a long term multi-criteria AC Transmission Expansion Planning model was developed considering two objectives - the probabilistic reliability index Expected Energy Not Supplied (EENS) and the investment cost. The Pareto-Front associated with these two objectives was obtained using Genetic Algorithms and the final solution was selected using a fuzzy decision making function. This approach was applied to the IEEE 24 Bus Test System and the results ensure its robustness and efficiency.
\end{abstract}

Index Terms - Multi-Criteria and Multi-Year Transmission Expansion Planning, Pareto-Front, Fuzzy Decision Making, EENS, AC-Optimal Power Flow.

\section{INTRODUCTION}

The main objective of the Transmission Expansion Planning (TEP) Problem is to define where, when and how a transmission system should be modified in order to adequately meet the future demand. This exercise can be conducted in order to consider several objective functions as minimizing the investment and operation costs, increasing the system reliability, minimizing the greenhouse gas emissions, increasing the flexibility of system operation while reducing the network charges, providing a better voltage profile, etc. Besides, the TEP problem can be modelled considering static or a dynamic (multiyear) approaches. In the first option, the study is performed considering each period at a time in a way that the equipments (transmission lines, cables or transformers) selected to expand the system in one period are considered on the basis topology for the subsequent ones. On the other hand, the multiyear approaches take the horizon in a holistic way and the problem is solved in a single run for all periods. It is noteworthy that the multiyear TEP preserves the holistic planning view and this is essential to obtain good quality long-term expansion plans. The experience of the authors also indicates that solving the TEP problem using a static year by year approach originates a global solution with a cost that is not inferior to the cost associated to the multiyear approach. This result is just the application of the well-known rule indicating that the aggregation of partial optima is not superior to the optimum coming from a global analysis.

One of the major concerns in Power Systems is surely related with their reliability, which in turn can be studied using deterministic or probabilistic approaches. Traditionally, TEP is conducted using the well-known deterministic " $\mathrm{N}-1$ " criterion. This criterion is applied based on worst-case analyses (draw from single contingency). However, this approach does not define consistently the true risk of the system, since it does not take into account how systems operates, how components fail and the existence of different load levels. In addition, the "N-1" criterion usually originates over-investments [1]. Differently, probabilistic approaches allow incorporating uncertainties associated to the non-ideal behavior of power system components. Meantime, the probabilistic approaches require pre-defining the reliability indices to be used, which in turn, may introduce some subjectivity in the analysis. As stated in [2], these aspects explain the dichotomy between deterministic and probabilistic approaches regarding the TEP problem.

In this paper a Multi-Criteria and Multi-Year Transmission Expansion Planning was developed considering two objectives - the investment cost and the probabilistic reliability index Expected Energy Not-Supplied (EENS). The developed approach uses a Non-Dominative CHA-Climbing Genetic Algorithm (NDCCGA) to build the Pareto-Front of the optimization problem and using this front it is then used a Fuzzy Decision Making Function (FDMF) to select the final expansion plan.

Regarding the structure of the paper, following this Introduction, Section II presents the AC model for the TEP problem and Section III provides a brief analysis of Reliability studies in power systems as well as the main steps of the chronological Monte Carlo Simulation to estimate the EENS index. Section IV details the NDCCGA tool and its main blocks, Section V presents a brief description of Fuzzy

The research leading to this work is funded by the CAPES under BEX number 13207-13-0. 
Decision Making and Section VI provides the results obtained in the simulations. Finally Section VII includes some comments and the conclusions about this work.

\section{TEP MATHEMATICAL FORMULATION}

The most adequate model to deal with TEP problems is the $\mathrm{AC}$ power flow (AC-OPF) operation model because it considers the reactive power, the losses and the bus voltage limits. However, this model is more demanding from a computational point of view than DC based models, thus requiring the use of very efficient optimization techniques to solve AC-OPF problems.

The AC-OPF used in this paper is formulated by (1) to (9).

$$
\begin{gathered}
\text { Min } C_{O P}=\sum \alpha_{i 1} P_{i}^{2}+\alpha_{i 2} \cdot P_{i}+\alpha_{i 3} \\
\text { subject to } \quad P(V, \theta, n)-P_{G}+P_{D}=0 \\
Q(V, \theta, n)-Q_{G}+Q_{D}=0 \\
P_{G \text { min }} \leq P_{G} \leq P_{G \text { max }} \\
Q_{G \text { min }} \leq Q_{G} \leq Q_{G \max } \\
V_{\text {min }} \leq V \leq V_{\text {max }} \\
(N+\stackrel{o}{N}) S^{\text {from }} \leq(N+\stackrel{o}{N}) S_{\text {max }} \\
(N+\stackrel{o}{N}) S^{t o} \leq(N+\stackrel{o}{N}) S_{\max } \\
0 \leq n \leq n_{\max }
\end{gathered}
$$

In this formulation $P(V, \theta, n)$ and $Q(V, \theta, n)$ are calculated by (10) and (11), and the bus conductance $\mathrm{G}$ and susceptance B are given by (12) and (13).

$$
\begin{gathered}
P(V, \theta, n)=V_{i} \sum V_{j}\left[G_{i j}(n) \cdot \cos \theta_{i j}+B_{i j}(n) \cdot \sin \theta_{i j}\right] \\
Q(V, \theta, n)=V_{i} \sum V_{j}\left[G_{i j}(n) \cdot \sin \theta_{i j}-B_{i j}(n) \cdot \cos \theta_{i j}\right] \\
G=\left\{\begin{array}{l}
G_{i j}(n)=-\left(n_{i j} \cdot g_{i j}+n_{i j} \cdot g_{i j}\right) \\
G_{i i}(n)=\sum_{j \in \Omega_{i}}\left(n_{i j} \cdot g_{i j}+n_{i j}\right. \\
o \\
B=\left\{\begin{array}{l}
o j \\
B_{i j}
\end{array}\right) \\
B_{i i j}(n)=b_{i j}^{s h}+\sum_{j \in \Omega_{i}}\left[n_{i j}\left(b_{i j}+b_{i j}^{s h}\right)+n_{i j}\left(b_{i j}+b_{i j}^{s h}\right)\right]
\end{array}\right\}
\end{gathered}
$$

The apparent flows $S_{i j}^{\text {from }}$ and $S_{i j}{ }^{t o}$ in branch ij are calculated by (14) and (15) where $P_{i j}^{\text {from }}, Q_{i j}^{\text {from }}, P_{i j}^{t o}$ and $Q_{i j}^{t o}$ are given by (16) to (19).

$$
\begin{gathered}
S_{i j}^{\text {from }}=\sqrt{\left(P_{i j}^{\text {from }}\right)^{2}+\left(Q_{i j}^{\text {from }}\right)^{2}} \\
S_{i j}^{\text {to }}=\sqrt{\left(P_{i j}^{t o}\right)^{2}+\left(Q_{i j}^{t o}\right)^{2}} \\
P_{i j}^{\text {from }}=V_{i}^{2} \cdot g_{i j}-V_{i} \cdot V_{j}\left(g_{i j} \cdot \cos \theta_{i j}+b_{i j} \cdot \sin \theta_{i j}\right) \\
Q_{i j}^{\text {from }}=-V_{i}^{2} \cdot\left(b_{i j}^{\text {sh }}+b_{i j}\right)-V_{i} \cdot V_{j}\left(g_{i j} \cdot \sin \theta_{i j}-b_{i j} \cdot \cos \theta_{i j}\right) \\
P_{i j}^{t o}=V_{j}^{2} \cdot g_{i j}-V_{i} \cdot V_{j}\left(g_{i j} \cdot \cos \theta_{i j}-b_{i j} \cdot \sin \theta_{i j}\right)
\end{gathered}
$$

$$
Q_{i j}^{t o}=-V_{j}^{2} \cdot\left(b_{i j}^{s h}+b_{i j}\right)+V_{i} \cdot V_{j}\left(g_{i j} \cdot \sin \theta_{i j}+b_{i j} \cdot \cos \theta_{i j}\right)
$$

In this formulation, the objective function (1) corresponds to the operation cost of a thermal system where $\alpha_{i 1}, \alpha_{i 2}$ and $\alpha_{i 3}$ are coefficients of the quadratic generator cost functions of each generation unit $i$ dispatching a real power $P_{i} . P_{G}$ is the real power generation, $Q_{G}$ is the reactive power generation, $P_{D}$ is the real power demand, $Q_{D}$ is the reactive power demand, $V$ is the voltage magnitude, $S_{i j}^{\text {from }}$ and $S_{i j}^{\text {to }}$ are the branch apparent flows in terminals, and $g_{i j}$ and $b_{i j}$ are the conductance and the susceptance of branch $\mathrm{i}-\mathrm{j}$.

\section{RELIABILITY IN POWER SYSTEMS}

\section{A. Overview on power system reliability methods}

In the assessment of power systems reliability, there are two different approaches that allow evaluating the adequacy of a power system and that can be used in TEP models: deterministic and probabilistic approaches. Deterministic criteria are based on a pre-specified rule that is defined considering the experience obtained through the analyses of other power systems. In TEP, the N-1 deterministic criterion to model likely failures is often taken in account. However, this approach does not consider the stochastic behaviour of power systems. This means that in order to consider uncertainties related to power systems such as the possible failure of system components, the weather conditions or the demand growth, a stochastic model should be followed. Markov processes are the well-known reference that allow including different system states. Figure 1 shows a typical two-state Markov model in which the failure and repair rates are modelled by exponential distributions. In other words, these distributions model the duration of the system events.

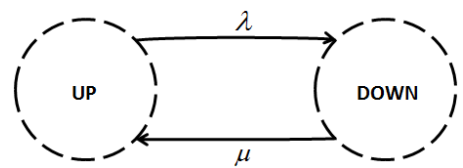

Figure 1: Markov model composed by two states, where $\lambda$ is the failure rate and $\mu$ is the repair rate.

Regarding the probabilistic approaches, there are two main families of methods that should be mentioned: the analytical and the simulation ones. The calculation of system reliability indices is the main goal of both approaches. However the application of analytical methods to complex power systems is not adequate due to the number of simplifications and assumptions that usually need to be accepted. The simulation processes are commonly known as Monte Carlo Simulations (MCS). MCS uses a random sampling of states in order to estimate the reliability indices. There are two different types of MCS: the chronological and the non-chronological. Since the developed TEP approach uses the EENS to characterize the system reliability, it is important to keep track of the sequence of states determining the life of the system together with their duration. Therefore, in this paper, the chronological MCS will be used to estimate the EENS index as it will be detailed in the next Section. 


\section{B. Chronological Monte Carlo Simulation to estimate the Expected Energy Not Supplied, EENS}

As mentioned before, the developed multiyear TEP problem includes the EENS to measure the reliability of the system under analysis. If just a non-chronological MCS was used, each system state was characterized by the on or off state of each component, an AC OPF could be run considering the on components and the associated Power Not Supplied could then be obtained. However, there would be no indication about the duration of each state in the sense that the operation-failure cycle of each component was not considered. In order to get more insight on system operation and to pass from PNS to ENS it is necessary to sample operation and repair times of the components thus justifying the use of a chronological Monte Carlo to estimate the EENS. The chronological or sequential MCS approach requires using the failure density function to model the operation and repair times of each component usually modeled by an exponential distribution [3]. The main blocks of the chronological MCS to evaluate reliability indices are presented below.

Procedure Chronological MCS

Initialize system data: MTTF, MTTR, pre-specified $\beta$

Do

Initialize the system state: through the selected probability distribution, sample the operating time of each system component.

Repeat

Pick the lowest sampled time. Let $\boldsymbol{F}$ be the associated component.

Evaluate the system state. According to this system state, calculate the power not supplied and then the energy not supplied multiplying the power not supplied by the duration of the current system state.

Update the accumulators of the reliability indices.

Depending on the previous state of component $F$, sample a new operating or repair time.

Update the state and the lifetime of $F$.

Evaluate the system lifetime. For each year of simulation, the reliability indices must be updated as well as the coefficient of variation $B$.

Until coefficient of variation $\beta$ or the maximum number of years is reached.

\section{End Chronological MCS}

The previous sequential MCS blocks show that for each system component an operation-repair life cycle is developed. Using this cycle, the energy not supplied can be calculated for each system state and consequently it is possible to obtain an estimate for EENS. This index reflects states in which the load is not fully supplied because it exceeds the available generation capacity and/or because there is insufficient branch transmission capacity.

\section{Non-Dominative Hill-Climbing Genetic ALGORITHM, NDCCGA}

The main blocks of the NDCCGA are similar to the ones of a genetic algorithm applied to solve the TEP problem. Additionally, it includes an improvement population block, a Tabu list to control the diversity of the population and a genetic similarity control to ensure the elite diversity through the generations. The main NDCCGA blocks are presented below and detailed in the next sections.

\section{Procedure NDCCGA}

Set the list of projects having nproj elements.

Initialize a random population with $p s$ individuals.

Repeat

Reproduction

Mutation

Improvement

Evaluation

Selection

Similarity Control

Stop Test

Until test is positive

\section{End NDCCGA}

\section{A. Possible projects - The Search Space Reduction}

The planner should specify a list of possible projects defined in terms of the extreme nodes, type (overhead line or transformer), transmission capacity and investment cost. The TEP algorithm should then select some of the elements in this list to be integrated in the expansion plan and locate them in one of the years of the horizon. The current approach restricts these projects to corridors already used in the base topology although it can be adapted to allow using new corridors. On the other hand, using a large number of branches can turn the computational effort to solve the TEP problem prohibitive. Therefore, the search space was reduced using a Constructive Heuristic Algorithm (CHA) detailed in [4].

\section{B. Particle Codification}

Each individual corresponds to an expansion solution plan and it is encoded by a vector that includes as many genes as the number of equipments in the reduced list coming from the CHA mentioned in IV.A. Each gene contains an integer number that represents the period of the planning horizon in which this equipment will be inserted into the network.

\section{Creating the Initial Population}

The initial population is randomly created with the aid of a Tabu List which ensures the diversity of this population.

\section{Reproduction}

In the reproduction block pairs of individuals randomly chosen are used to create an offspring. Differently from usual crossover strategies in which just one position in each 
individual is sampled, in this case we sample two positions. Starting with the first individual we sample another one to form a pair, then two positions are sampled in these two individuals and one offspring is created. This procedure is repeated until all individuals undergo reproduction thus creating a new population with the same size as the initial one.

\section{E. Mutation}

The mutation only affects a small percentage of the offsprings and it aims at increasing the diversity of a particular individual. Once an individual is selected, a particular gene is randomly sampled and then its associated integer number is modified. If this integer number is increased, than this means postponing the construction of the associated equipment.

\section{F. Improvement Block}

The improvement block is based on the individual's characteristics, that is, if an individual has an unacceptable value for PNS for a particular year, it is improved inserting new equipments selected using a CHA. On the other hand, if this individual displays a PNS value below a threshold, it is modified by eliminating equipments using the Hill Climbing Method. According to this method, an equipment is removed and the new individual is evaluated again. If the resulting PNS value continues below the threshold this change is confirmed. If not, that equipment is included back in the individual. These algorithms are detailed in [5] and they are used as a way to accelerate the convergence of the genetic algorithm reducing the investment cost or the EENS index of some solutions.

\section{G. Evaluation}

In the evaluation block the values of investment cost and EENS are calculated. In a first moment, the PNS is obtained by running the AC-OPF using (1) to (9). If this value is greater than zero the fitness function (20) is calculated and the EENS is set at $\beta_{2}$ (high value). If the PNS is zero the EENS is obtained as described in Section III. In (20) $\mathrm{p}$ is the period in study, $n p$ is the total number of periods, $r$ is the return rate and $\beta_{1}$ is the penalty factor for PNS.

$$
\text { fitness }=\sum_{p=1}^{n p} \frac{C_{i n v, p}}{(1+r)^{p}}+\beta_{1} . P N S
$$

\section{H. Selection}

The selection is performed in two steps as follows. In the first place, the non-dominated solutions are selected, regarding the objectives investment costs and EENS, and included in the new population. In the second step it is used a tournament selection with the remaining solutions to complete the population until its original size is reached.

\section{Genetic Similarity Control}

The non-dominated solutions coming from the first step of the Selection process are subjected to a similarity control to increase the diversity of the new population. This control uses the concept of parent circle of radius $r$ as illustrated in Figure 2. For each non-dominated solution a parental circle is used around it. If another solution is located inside this circle, then the solution having the highest investment cost is discarded.

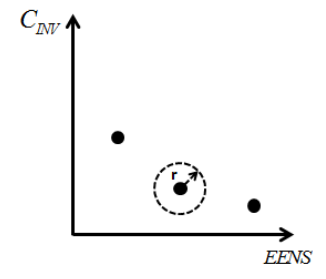

Figure 2: Illustration of the concept of parental circle.

\section{J. Stopping Criterion}

The stopping criterion is based on the set of non-dominant solutions. If this set remains unchanged for a pre specified number of iterations the iterative process ends.

\section{FUZZY DECISION MAKING}

Once the Pareto-front is built, the decision maker has a number of solutions characterized by the corresponding investment cost and EENS. In order to help the decision maker to select the final solution we used a fuzzy decision making function according to which the membership function $\mu_{i}$ measures the adequacy of each solution $\mathrm{k}$ in the Pareto front regarding a specific objective function for each $\mathrm{i}$ ( $f_{1}=C_{I N V}$ and $f_{2}=E E N S$ ). Figure 3 illustrates the concept associated with this approach. For each objective function i, $f_{i}$ , the decision maker specifies a minimum and a maximum level, so that if a solution $\mathrm{k}$ has a value for $f_{i}$ less than the minimum, then a 1,0 membership degree is assigned. If the value of $f_{i}$ is between the minimum and the maximum then a membership degree decreasing from 1,0 to 0,0 is associated indicating that solution $\mathrm{k}$ is less compatible with the concept of being a good quality solution in the sense of minimizing the objective $f_{i}$. Finally, if the value of $f_{i}$ is larger than the maximum level then the solution $\mathrm{k}$ has bad quality and it is eliminated. Once having the membership degrees regarding the two objectives for a solution $\mathrm{k}$, then the minimum of the two is taken and this value is used to characterize solution $\mathrm{k}$. Among all the solutions in the Pareto Front, it will be selected the one having the maximum of the minimum values of the corresponding objectives as it is translated by (20).

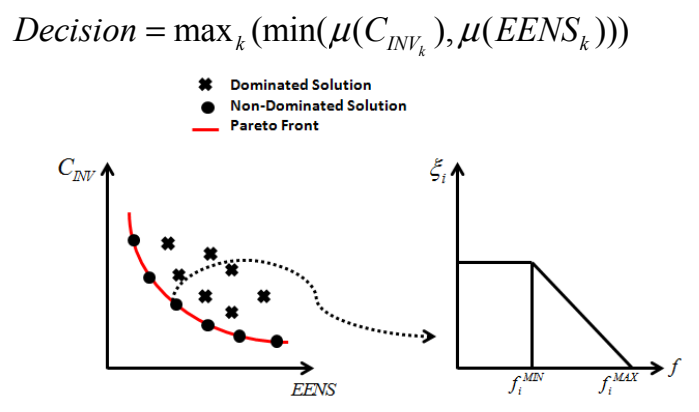

Figure 3: Fuzzy Mechanism for Best Comparison

\section{TESTS AND RESUlts}

The Non-Dominative CHA-Climbing Genetic Algorithm described in Section IV was applied on the modified IEEE 24 Bus Reliability Test System. The system used in the tests has some differences regarding the original system proposed in [6] and the system details can be found in [3] and [4]. 
The tests were performed considering the multiyear TEP model with 3 periods and using a load increase of 5\% per period. The original list of equipments includes 38 lines and transformers and in the first place the CHA was applied to select a sub-list of candidate equipments considering a static TEP problem for each period. After solving 23 AC-OPFs, using the MATPOWER tool described in [7], running in MATLAB with an Intel i7, 3.4GHz, 8 GB RAM, the CHA selected 10 branches for possible reinforcement from the initial 38 equipments leading to a reduction of $99 \%$ in the search space. The equipments that result from search space reduction step are the ones connecting buses 1-5, 3-24, 6-10, $7-8,11-13,13-23,14-16,15-24,16-17,17-18$, in which it is permitted build up to 3 circuits for each path.

The simulations were performed in MATLAB with an Intel i7, 3.4GHz, $8 \mathrm{~GB}$ RAM. The NDCCGA used 50 individuals in the population and the parent circle radius was set at $10^{4}$. The parameters for the CHA and the Hill Climbing methods are the same as used in [5]. The simulation involved solving about 1200000 AC-OPFs in about 100 hours. Figure 4 shows the solutions obtained, in which the vertical axis corresponds to the present value of the investment cost, that is, the sum of the investment cost in the three periods brought back to the departing period using a return rate of 5\% per year. The horizontal represents the EENS for the three years.

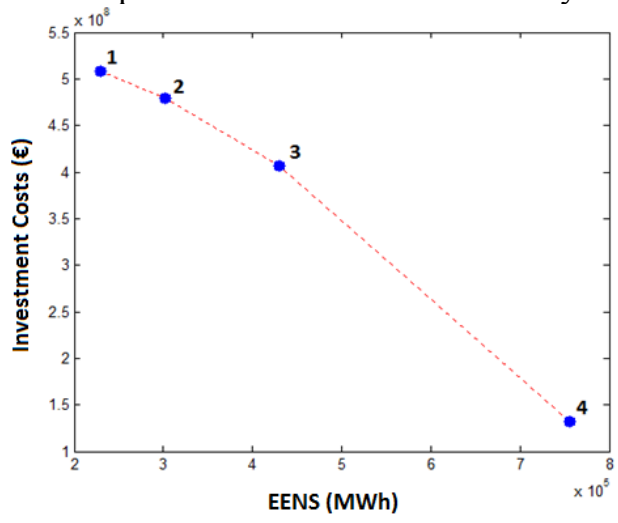

Figure 4: Pareto-Front associated with the investment cost and EENS.

The shape of this front deserves a comment because it behaves differently regarding the theoretical illustration in Figure 3. The TEP problem has a discrete nature so that including a new equipment in the expansion plan or changing the set of equipments to pass, for instance, from solution 4 to solution 3 leads to discrete jump in the investment cost. Apart from that, the evaluation of EENS requires solving non-linear AC problems. Together, these two issues determine that a convex shape has the one in Figure 3 is not always obtained in the case of the TEP problem.

TABLE I. BEST SOLUTION IDENTIFIED BY THE FUZZY DECISION MAKING

\begin{tabular}{|c|c|c|c|}
\hline Period & New equipment & $\begin{array}{c}\text { Invest. } \\
\text { Cost }(€)\end{array}$ & EENS (MWh) \\
\hline 1 & $1-5,6-10,7-8,11-13$ & $120.10^{6}$ & $46,2 \cdot 10^{3}$ \\
\hline 2 & $7-8,11-13,(2) 14-16,15-24$ & $262.10^{6}$ & $205,43.10^{3}$ \\
\hline 3 & $1-5,3-24,16-17$ & $108.10^{6}$ & $178,87.10^{3}$ \\
\hline
\end{tabular}

After building the Pareto-Front, it was used the Fuzzy Decision Making described in Section V. For each Pareto Front solution $\mathrm{k}$, it was calculated the membership function given by (20). The final solution corresponds to solution 3 in Figure 4 and detailed in Table I.

\section{CONCLUSIONS}

This paper presents a dynamic approach of the Transmission Expansion Planning problem using a multicriteria analysis that considers the total investment cost and the Expected Energy Not Supplied as the objectives. The problem was solved using the Non-Dominative CHAClimbing Genetic Algorithm tool developed by Vilaça and Saraiva in [5]. A Fuzzy decision making process is then used to select the final expansion plan among the solutions in the Pareto Front. The EENS index was estimated using a Chronological Monte Carlo Simulation.

The NDCCGA was applied to the modified IEEE 24-Bus Reliability Test System and showed excellent performance to deal with the large computational effort required to estimate the EENS and to solve the AC-OPFs. The tool provided a set of 4 final solutions with different values of investment cost and EENS. On the other hand, the adoption of multi-objective approaches is very relevant as a way to turn the TEP problem more realistic. It also offers a trade-off analysis between objectives instead of other techniques that, for instance, require transforming all objectives except one in constraints or building a value function that aggregates all the individual objectives using weights specified by the user.

As future work, the developed toll can be parallelized to take advantage of multi-core machines.

\section{ACKNOWLEDGMENT}

P. Vilaça acknowledges the financial support from CAPES Scholarship under Bex 13207-13-0.

\section{REFERENCES}

[1] J. P. V. Vasques, "Definition of maintenance policies in power systems," M.Sc Thesis, Department of Electrical and Computer Engineering - University of Porto, 2014.

[2] J. R. P. Barros, A. C. G. Melo, and A. M. L. da Silva, "An approach to the explicit consideration of unreliability costs in transmission expansion planning," in 2004 IEEE 11th International Conference on Probabilistic Methods Applied to Power Systems, 2004, pp. 927-932.

[3] R. Billinton, Reliability Evaluation of Engineering Systems Concepts and Techniques. New York, 1983.

[4] P. V. Gomes and J. T. Saraiva, "Hybrid Discrete Evolutionary PSO for AC Dynamic Transmission Expansion Planning," in IEEE Energycon conference, 2016.

[5] P. V. Gomes and J. T. Saraiva, "Hybrid Genetic Algorithm for Multi-Objective Transmission Expansion Planning," in IEEE Energycon conference, 2016.

[6] P. Subcommittee, "IEEE Reliability Test System," IEEE Trans. Power Appar. Syst., vol. PAS-98, no. 6, pp. 2047-2054, Nov. 1979.

[7] R. D. Zimmerman, C. E. Murillo-Sanchez, and R. J. Thomas, "MATPOWER: Steady-State Operations, Planning, and Analysis Tools for Power Systems Research and Education," IEEE Trans. Power Syst., vol. 26, no. 1, pp. 12-19, Feb. 2011. 